\title{
AUTOMATIC FAULT RECOGNITION OF PHOTOVOLTAIC MODULES BASED ON STATISTICAL ANALYSIS OF UAV THERMOGRAPHY
}

\author{
Dusik Kim*, Junhee Youn and Changyoon Kim
}

Korea Institute of Civil Engineering and Building Technology (KICT), Goyangdae-Ro, Ilsanseo-Gu, Goyang-Si, Gyeonggi-Do,

Republic of Korea - (dusikkim, younj, ckim)@kict.re.kr

KEY WORDS: Photovoltaic Module, UAV Inspection, Fault Diagnosis, Infrared Thermography

\begin{abstract}
:
As a malfunctioning PV (Photovoltaic) cell has a higher temperature than adjacent normal cells, we can detect it easily with a thermal infrared sensor. However, it will be a time-consuming way to inspect large-scale PV power plants by a hand-held thermal infrared sensor. This paper presents an algorithm for automatically detecting defective PV panels using images captured with a thermal imaging camera from an UAV (unmanned aerial vehicle). The proposed algorithm uses statistical analysis of thermal intensity (surface temperature) characteristics of each PV module to verify the mean intensity and standard deviation of each panel as parameters for fault diagnosis. One of the characteristics of thermal infrared imaging is that the larger the distance between sensor and target, the lower the measured temperature of the object. Consequently, a global detection rule using the mean intensity of all panels in the fault detection algorithm is not applicable. Therefore, a local detection rule based on the mean intensity and standard deviation range was developed to detect defective PV modules from individual array automatically. The performance of the proposed algorithm was tested on three sample images; this verified a detection accuracy of defective panels of $97 \%$ or higher. In addition, as the proposed algorithm can adjust the range of threshold values for judging malfunction at the array level, the local detection rule is considered better suited for highly sensitive fault detection compared to a global detection rule.
\end{abstract}

\section{INTRODUCTION}

The international community agreed to pursue efforts to limit the global average temperature increase to well below $1.5^{\circ} \mathrm{C}$ as a part of the Paris Agreement in December 2015. The primary methodology of holding global temperature increase is restriction of carbon gas emissions. Therefore, eco-friendly electricity generation method such as solar and wind became alternative strategies for securing future energy and the sustainable environment.

PV (photovoltaic) power generation systems have received significant attention as one of the promising renewable energy sources. However, the PV panels of solar power plants must installed outdoors to collect radiation energy. It means the PV module can be affected by various environmental sources such as wind, salt, snow and dust. These factors may cause the corrosion, short circuit and decrease of generating efficiency of the PV module. Therefore, regular fault inspection and constant maintenance and repair are essential for maintaining a stable performance of PV systems.

Visual inspection and output measurement methods can be used for fault diagnosis in PV panels with reduced output efficiency (Quater et al., 2014). However, most of the PV power plants placed in-line and series connected. Therefore, individual PV module inspection is hard with this inspection method in largescale PV power plant. During PV module operation, the temperatures of defective cells are higher than the normal cells. The temperature difference appear as hot spots in thermal infrared image of a PV module and people can recognize them obviously. However, it will be a time-consuming way to inspect large-scale PV power plants by a hand-held thermal infrared sensor.

Recently, a PV power plant monitoring technology has been developed that uses UAVs equipped with thermal imaging cameras (Buerhop and Scheuerpflug, 2014; Grimaccia et al., 2015). This is a promising technology for monitoring largescale PV power plants as it can rapidly scan a large PV array field. At the current level of technology, however, detection of defective panels is through the visual assessment of the images captured by aerial photogrammetry, and the analysis of a large number of image frames is time-consuming. This can be addressed only by developing a method for automated fault detection by combining aerial photogrammetry with computer vision technology.

Two core technologies are involved in the process of automated detection of defective panels of a PV power plant from aerial thermal infrared images. The first one is technology for automatically extracting the ROI (region of interest) of a PV array field from the given images. Tsanakas et al. (2015) and Rogotis et al. (2014) presented methods for extracting the ROI from terrestrial thermal infrared image sequences using the Canny edge operator (Canny, 1986) or image segmentation techniques (Gonzalez et al., 2004). More recently, Kim et al. (2016a, 2016b) proposed an algorithm for panel area extraction from thermal infrared images captured with a UAV using the Canny edge operator and image segmentation techniques. They concluded that the area extraction method using the Canny edge operator did not lend itself well to creating a single polygon for the panel area due to noises within and outside the panels. In contrast, the image segmentation-based area extraction method

\footnotetext{
* Corresponding author

This paper is revised version of a paper presented at the Journal of the Korean Society of Surveying, Geodesy, Photogrammetry and Cartography Vol. 34 , No. 6
} 
was able to create polygons of individual panel areas, albeit limited by imperfect linearity.

The second core technology is one enabling automatic diagnosis of defective panels based on extracted PV panel areas. Tsanakas et al. (2015) designed a method to identify the location of hot spot cells on a PV panel using the Canny edge operator. In the PV power plant maintenance and repair regime generally applied in South Korea, any panel containing defective cells is replaced in its entirety. Hence, the algorithm should focus on diagnosing the function or malfunction of each panel rather than identifying the locations of hot spot cells within a panel. Consequently, it is considered reasonable to employ a method for comparing the intensity characteristics of individual panel area polygons, rather than one using threshold values such as the Canny edge operator, for developing an algorithm in this study. In a related previous study, Kim et al. (2016a) proposed using statistical characteristics of thermal intensity as parameters for panel fault diagnosis because defective panels display different patterns of intensity compared to intact panels. Drawing on this finding, this study aims to develop an algorithm capable of automated PV panel fault diagnosis using intensity-related statistical values of each panel based on extracted panel area polygons.

\section{ANALYSIS OF PV PANEL INTENSITY CHARACTERISTICS IN A THERMAL INFRARED IMAGE}

As experimental data, we used a sample image (Figure 1 (a)) obtained from a UAV with FLIR T620 thermal imaging camera), provided online by paul kitawa at a pixel size of $640 \times 480$. In a previous study (Kim et al., 2016b), we developed an image segmentation-based panel area extraction algorithm and created polygons for individual panel areas as shown in Figure 1 (b). The study confirmed that panel areas could be expressed with 93.9\% accuracy compared with visually extracted and manually digitized panel boundaries using the performance assessment method of McGlone et al. (2004). In Figure 1 (c), each panel is labelled with a serial number assigned to each extracted panel area polygon. Visual inspection of the labelled panels reveals that hot spots are present on a total of five panels $(6,23,26,64$, and 66) a string form. Analysis of the thermal characteristics of defective PV modules suggests these hot spots are due to defective bypass diodes within the modules.

To reduce fault diagnosis error, it is essential to consider the overall intensity characteristics of each panel. We compared intensity histograms in order to determine the intensity characteristics of normal and defective panels (Figure 2).
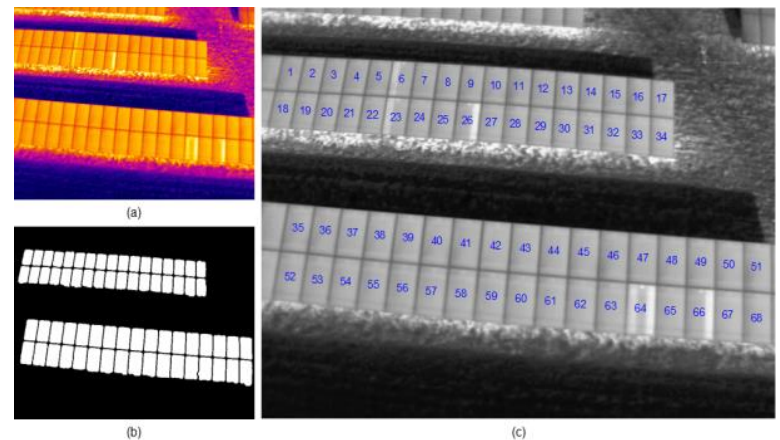

Figure 1. (a) Sample thermal infrared image (www.kiwata.de), (b) Panel area extraction result, and (c) Grayscale image with PV module numbers labelled

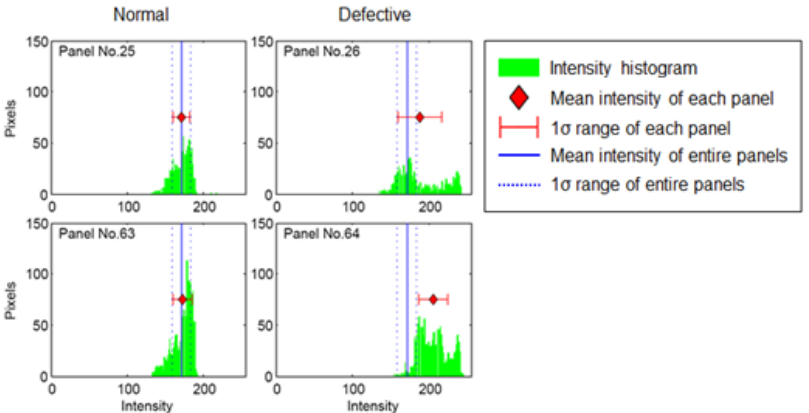

Figure 2. Examples of intensity histogram and statistical characteristics of normal and defective panels

The histograms on the left side in Figure 2 represent normal panels (25 and 63), and those on the right side represent defective panels (26 and 64). Because intensity distributions of normal panels are similar to those of adjacent panels, normal panels close to panels with hot spots were selected for comparison. The blue solid and dotted lines in Figure 2 represent the mean intensity and standard deviation range, respectively, of pixels in the entire panel area. The red diamond and solid line represent the mean intensity and standard deviation range, respectively, of the pixels in each panel area concerned. The normal panel histograms are distributed at an intensity range of 200 or lower and display patterns similar to the normal distribution of the entire panel area. In contrast, the defective panel histograms contain pixels with intensity values exceeding 200 and display multiple peaks that deviate from a normal distribution. Comparison of the mean intensity and standard deviation range of each panel revealed that while normal panels displayed values similar to the standard deviation range of the entire panel area, defective panels displayed higher values. This result is attributable to the increased intensity distribution range caused by the hot spots present on defective panels, resulting in a larger standard deviation.

\section{DEVELOPMENT AND EVALUATION OF THE FAULT DIAGNOSIS ALGORITHM}

From the analysis of intensity-related statistics, we can derive a method for assigning a reference value applicable to the fault diagnosis algorithm. For the purpose of this study, the mean intensity and standard deviation of each panel were selected as parameters for fault diagnosis of PV panels. First, any panel with a mean intensity that deviates from the sample standard deviation of the sample mean intensities of the adjacent sample panels can be defined as a candidate defective panel. Here, adjacent panels are selected from panels situated in the same array. If the intensities of each panel can be normalized, the malfunction panel can be detected by analysing the intensities of the entire panel. However, for the normalization of the intensities, parameters that can grasp the geometrical relationship between the target and the sensor are required. Since these parameters were not obtained in this study, a comparison method between adjacent panels (panels located in the same array row) was applied to detect malfunctioning panels with only sample images. As such, if the standard deviation is calculated after dividing the number of the sample panels, the reference value for diagnosing a defective panel can be adjusted for each array, and candidate defective panels can be detected efficiently. In this study, this is defined as the CMI (criterion for mean intensities). Next, any panel with a $1 \sigma$ intensity range larger than the standard deviation (average range of samples standard deviations) of all the adjacent panels can be classified 
as a candidate defective panel. In this paper, this is defined as the CSD (criterion for standard deviations). Here, the average standard deviation of all the adjacent panels was calculated by assigning weight to the number of pixels using Eq. (1), in order to reflect the different number of pixels in each panel ROI.

$$
S_{w}=\sqrt{\frac{\left(n_{1}-1\right) S_{1}^{2}+\left(n_{2}-1\right) S_{2}^{2}+\cdots+\left(n_{k}-1\right) S_{k}^{2}}{n_{1}+n_{2}+\cdots+n_{k}-k}}
$$

where, $S_{w}=$ weighted average of standard deviations

$$
\begin{aligned}
& n=\text { number of pixels of each panel } \\
& S_{x}=\text { standard deviation of each panel } \\
& k=\text { number of panels. }
\end{aligned}
$$

The conditions for defective panel detection were set as follows: (i) a mean panel intensity larger than the CMI; (ii) the sum of the average of standard deviations and mean intensity of the target panel larger than the sum of the CMI and mean intensity. These detection conditions were selected because the purpose of this study is to detect defective panels under the assumption that panels with hot spots are defective panels. Consequently, a panel with a mean intensity lower than the CMI range on the image is considered a normal panel. This criterion setting method, which uses $1 \sigma$ range, is an empirical method derived from thermal infrared image analysis of solar power plants. Since this method based on the empirical characteristics of intensity, various sample image analysis should be added to improve the integrity of the algorithm. Figure 3 describes flow chart of fault diagnosis algorithm.

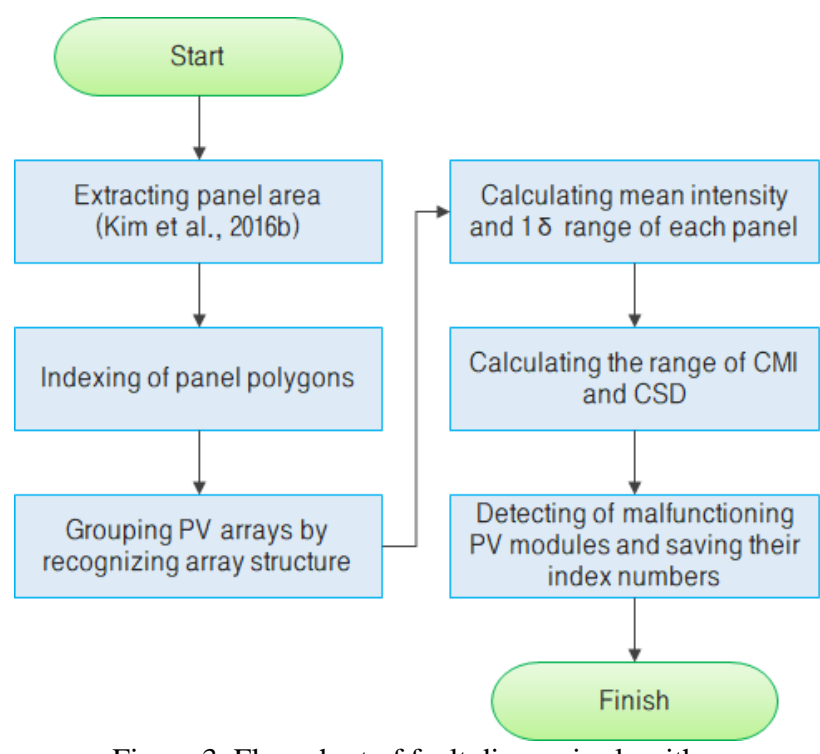

Figure 3. Flow chart of fault diagnosis algorithm

Figure 4 presents results of diagnosing defective panels using the proposed algorithm. Red and green squares represent the mean intensities of normal panels and panels diagnosed as defective, respectively. Red lines represent the $1 \sigma$ range of a given panel. Blue + and red $\times$ represent the CMI and CSD ranges, respectively. In order to show whether a panel classified as a defective panel met the CSD condition, the CSD range of the panel concerned was marked as a blue $\times$. Figure 4 shows that a total of seven panels were recognized as defective, although the actual number of defective panels with hot spots on the image was confirmed as five. Hence, the algorithm was found to have limitations in fault diagnosis by classifying panels without hot spots, such as panels 17 and 50, as defective panels.

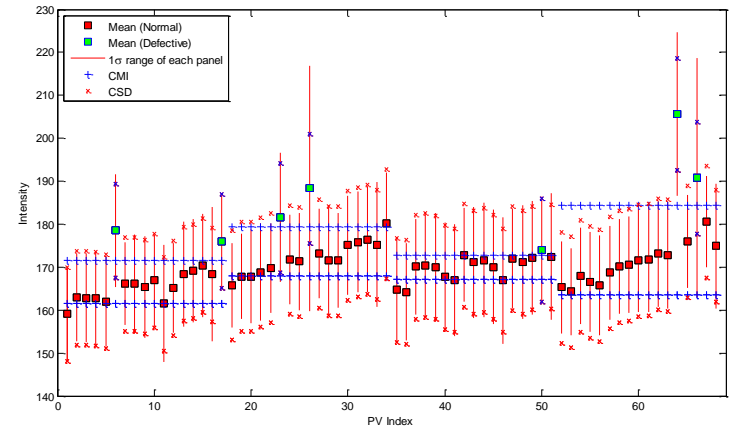

Figure 4. Result of fault diagnosis algorithm application

We performed additional algorithm implementation on two images for the purpose of evaluating the proposed algorithm. Figure 5 (a) and (b) present the results of target panel labelling after converting two thermal infrared images of $640 \times 480$ pixel size into grayscale images. The images were retrieved from the website of the paul kitawa company, as the example image presented earlier. Drawing on our previous study (Kim et al., 2016 b), in which panel area ROIs were generated from these images, we were able to evaluate the results of algorithm implementation using the images and ROI data. There are three defective panels $(11,12$, and 13) on image (a) and five defective panels $(4,32,59,60$, and 65) on image (b). Figure 5 (c) and (d) present the results of panel area extraction.

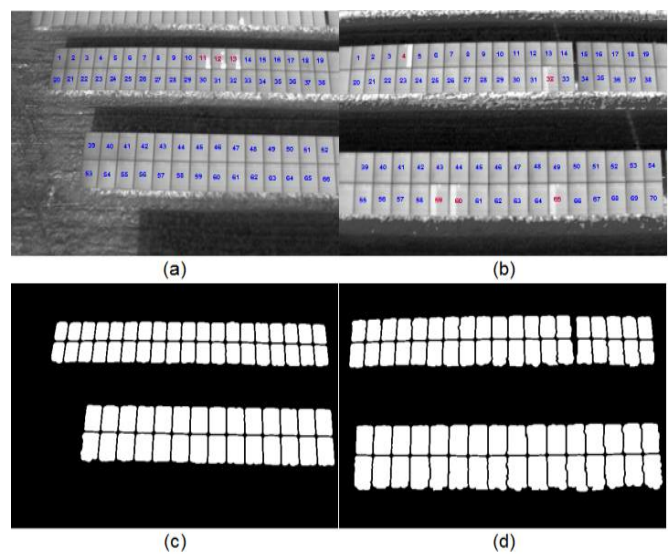

Figure 5. (a) and (b) Additional test images for evaluation of the fault diagnosis algorithm (www.kitawa.de), (c) and (d) Results of panel area extraction

Figure 6 and 7 represent the results of algorithm implementation to the images in Figure 5 (a) and (b), respectively. The results of algorithm implementation on the two images were consistent with the defective panels recognized by visual inspection, and no diagnosis error of recognizing a defective panel and a normal panel occurred. In Figure 6, the mean intensity of each panel was found to increase exponentially as the row in the same array advanced. Likewise, the mean intensities of normal panels generally showed a continuous pattern well-aligned with the adjacent panels in terms of mean intensity. These characteristics allowed us to conclude that it was adequate to select the mean intensity of each panel as the main parameter for fault diagnosis in the proposed algorithm. Table 1 outlines the results of performance evaluation using a confusion matrix on the basis of the number of panels inspected. The proposed algorithm recognized all defective panels displayed on the images. As presented in Table $1, \mathrm{FN}$ is 0 in all three images, i.e., completeness is $100 \%$, which means that the proposed algorithm recognized all defective 
panels displayed on the images. As such, this algorithm has met the purpose of detecting defective panels. However, the algorithm achieved an accuracy of approximately $97 \%$ in Fig. 1, which implies that a normal panel might be recognized as a defective panel. Therefore, it is considered necessary to improve the accuracy of the algorithm by setting out additional conditions in a future study.

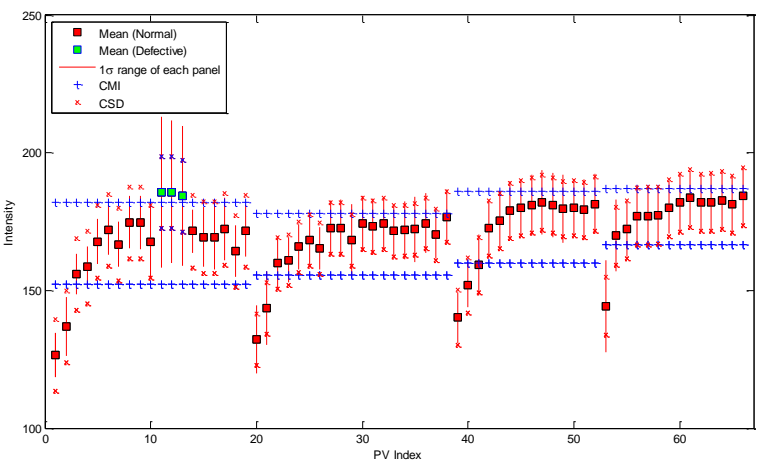

Figure 6. Result of algorithm implementation to the image in Figure 5 (a)

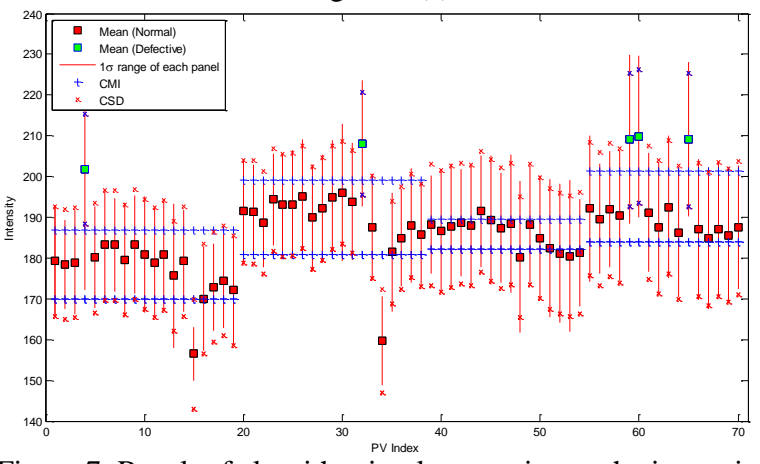

Figure 7. Result of algorithm implementation to the image in Figure 5 (b)

Table 1. Comparison of fault detection accuracies

\begin{tabular}{c|c|c|c|c|c|c}
\hline \hline \multirow{2}{*}{ Image } & TP & FN & FP & TN & Completeness & Accuracy \\
\cline { 2 - 7 } & \multicolumn{3}{|c|}{ [No. of Panels] } & \multicolumn{2}{c}{ [\%] } \\
\hline Fig. 1 & 5 & 0 & 2 & 61 & $100 \%$ & $97.06 \%$ \\
\hline Fig. 5 (a) & 3 & 0 & 0 & 63 & $100 \%$ & $100 \%$ \\
\hline Fig. 5 (b) & 5 & 0 & 0 & 65 & $100 \%$ & $100 \%$ \\
\hline
\end{tabular}

\section{CONCLUSIONS}

We developed an algorithm capable of automatically diagnosing defective PV panels based on intensity statistics. As parameters for fault diagnosis, we selected mean intensity and standard deviation range, and applied a local detection rule to diagnose faults using the statistics of each array row, not of the entire panels. In comparison with the general detection rule of computing the standard deviation of the entire data range, this method is judged to be better suited for sensitive classification because it can narrow the range of determining normal or defective panels. However, given that the proposed algorithm was implemented only on three thermal infrared images in this study, additional image analysis will have to be performed to prove the reliability of the algorithm in terms of completeness and accuracy and further improve its performance. Additionally, the sample images were of similar panel sizes and intensity characteristics; it is thus necessary to conduct a further sample analysis study, thereby varying conditions such as the years of service of panels, observation hours, and scale of photogrammetric measurement. Despite the small sample size analyzed, this study is significant in that it ascertained the feasibility of using intensity statistics of thermal infrared images as parameters for automatic fault diagnosis of PV panels. In future research, it is considered necessary to explore how to use orientation parameters of a sensor and ground control point (GCP)-based photogrammetric survey to precisely determine the locations of defective panels to establish an efficient maintenance and repair regimen for large-scale PV power plants.

\section{ACKNOWLEDGEMENTS}

This research was supported by Basic Science Research Program through the National Research Foundation of Korea (NRF) funded by Ministry of Science, ICT and Future Planning and Ministry of Education (NRF-2017R1D1A1B06035079).

\section{REFERENCES}

Buerhop, C. and Scheuerpflug, H., 2014. Field inspection of PV-modules using aerial, drone-mounted thermography. In: The 29th European Photovoltaic Solar Energy Conference and Exhibition (EUPVSEC), Amsterdam, Netherlands, pp. 29752979.

Canny, J., 1986. A computational approach to edge detection. IEEE Transactions on Pattern Analysis and Machine Intelligence (PAMI), Vol. 8, No. 6, pp. 679-698.

Gonzalez, R.C., Woods, R.E., and Eddins, S.L., 2004. Digital Image Processing using Matlab. Pearson Prentice Hall, N.J.

Grimaccia, F., Aghaei, M., Mussetta, M., Leva, S., and Quater, P.B., 2015. Planning for PV plant performance monitoring by means of unmanned aerial systems (UAS). International Journal of Energy and Environmental Engineering, Vol. 6, No. 1, pp. 47-54.

Kim, D., Youn, J., and Kim, C., 2016a. Determination of Defective Photovoltaic Module by using Thermography. In: The 37th Asian Conference on Remote Sensing (ACRS), Colombo, Sri Lanka.

Kim, D., Youn, J., and Kim, C., 2016b. Automatic Photovoltaic Panel Area Extraction from UAV Thermal Infrared Images. Journal of the Korean Society of Surveying, Geodesy, Photogrammetry and Cartography, Vol. 34, No. 6, pp. 559-568.

McGlone, J.C., Mikhail, E.M., and Bethel, J.S., 2004. Manual of Photogrammetry. American Society of Photogrammetry and Remote Sensing, pp. 969-970.

Quater, P.B., Grimaccia, F., Leva, S., Mussetta, M., and Aghaei, M., 2014. Light Unmanned Aerial Vehicles (UAVs) for Cooperative Inspection of PV Plants. IEEE Journal of Photovoltaics, Vol. 4, No. 4, pp. 1107-1113.

Rogotis, S., Ioannidis, D., Tsolakis, A., Tzovaras, D., and Likothanassis, S., 2014. Early defect diagnosis in installed PV modules exploiting spatio-temporal information from thermal images. In: The 12th Quantitative InfraRed Thermography Conference (QIRT), Bordeaux, France.

Tsanakas, J.A., Chrysostomou, D., Botsaris, P.N., and Gasteratos, A., 2015. Fault diagnosis of photovoltaic modules through image processing and Canny edge detection on field thermographic measurements. International Journal of Sustainable Energy, Vol. 34, No. 6, pp. 351-372. 